\title{
Effects of Solar Radiation on the Performance of Pyrgeometers with Silicon Domes
}

\author{
L. AlaDOS-ARBoledAS, J. VidA AND J. I. JiMÉNEZ \\ Departamento de Fisica Aplicada, Facultad de Ciencias, Universidad de Granada, Granada, Spain \\ 6 August 1987 and 8 January 1988
}

\begin{abstract}
The performance of the Eppley pyrgeometer with a silicon dome presents several anomalies during daytime measurements. These problems are related mainly to the solar heating of the dome, which causes nearly instantaneous fluctuations, about $\pm 1 \%-2 \%$ from the average value, in the longwave irradiance measured by the pyrgeometer during cloudless days.

An evaluation of the solar heating effect of the dome made with a set of sun and shade experiments indicates that the pyrgeometer output includes an equivalent of about $3.6 \%$ of the incoming solar radiation. In spite of the improvement with the use of silicon instead of KRS-5 domes, the error due to solar heating requires some kind of correction. Sun and shade experiments under a number of wind speed conditions are used to derive an empirical correction that is based on the global solar radiation.
\end{abstract}

\section{Introduction}

The Eppley pyrgeometer is an instrument designed to directly measure thermal atmospheric irradiance. For this reason it incorporates a silicon dome with an interference filter that isolates the infrared (4-50 $\mu \mathrm{m})$ portion of the spectrum. This instrument is a modified version of the former Eppley pyrgeometer that included a KRS-5 (binary thallium iodide-thallium bromide) dome.

Several authors (Albrecht et al. 1974, 1977; Enz et al. 1975) have studied the problems associated with the performance of the former KRS-5 pyrgeometer. In addition to the problem associated with the instrument circuitry studied by Albrecht and Cox (1977), the KRS5 pyrgeometer presented serious problems during daytime measurements. Enz et al. (1975) found nearly instantaneous fluctuations of $\pm 2 \%-5 \%$ about the average value on clear days, while during clear nights the maximum short-term variations in measured longwave irradiance were about $\pm 0.5 \%$ of the average value. These authors also pointed out an abnormal correlation between pyrgeometer measurements and solar radiation.

Although the silicon dome pyrgeometer represents an improvement over the KRS-5 model, it is not completely free from the limitations mentioned above. Thus, tests on the new model, carried out as part of the present investigation, showed the existence of fluctuations in the instrument output during cloudless days, although the magnitude of this effect is considerably reduced (down to a maximum value of $\pm 1 \%$ -

Corresponding author address: Dr. J. 1. Jiménez, Departamento de Fisica Aplicada, Universidad de Granada, 18701-Granada, Spain.
$2 \%$ ). The abnormal correlation between measurements and radiation was also detected. According to Berdahl and Fromberg (1982) this correlation is not explained by direct transmission of solar radiation through the silicon dome, but seems to be due to solar heating of the dome (either KRS-5 or silicon) that results in additional energy fluxes over the sensor surface.

In this paper we present the results of several experiments designed to evaluate the performance of the silicon dome pyrgeometer in daytime. Our calculation of the energy budget over the sensor surface indicates that about $11-12 \mathrm{~W} \mathrm{~m}^{-2}$ are added to the pyrgeometer measurements for each degree of difference between dome and base temperatures.

\section{Instrumentation}

The experiments shown in this study were carried out in the radiometric station of the University of Granada, located on the rooftop of the physics building in the Faculty of Sciences $\left(37^{\circ} 11 \mathrm{~N}, 3^{\circ} 35 \mathrm{~W}, 680 \mathrm{~m}\right.$ above MSL).

Thermal atmospheric irradiance was measured with an Eppley precision infrared radiometer (pyrgeometer), model PIR. This instrument employs a silicon dome with an interference filter deposited on its inner surface. The dome exhibits a transmissivity of about 0.5 for the wavelength interval $4-50 \mu \mathrm{m}$ and is opaque for wavelengths lower than $3 \mu \mathrm{m}$ (Eppley 1976).

Our radiometric station includes some instruments for solar radiation measurements. The global solar radiation is measured with a Kipp-Zonnen pyranometer (Model CM-11), and a Middleton pyrheliometer (Model CN-5), used for measuring the direct solar radiation. Other parameters measured at the station in- 
clude diffuse solar radiation and temperature and relative humidity at screen level.

For our particular experiments, described below, the measurements of interest were those concerning atmospheric thermal irradiance and direct solar radiation at normal incidence.

\section{Pyrgeometer performance}

The energy budget on the sensor surface is summarized in the Appendix. If the dome and pyrgeometer base have the same temperature, the pyrgeometer performance is well explained by

$$
L=\left(c_{1}+c_{2} T_{0}^{3}\right) V+\sigma T_{0}^{4}
$$

where $L$ indicates the thermal atmospheric irradiance and the other symbols are explained in the Appendix. The pyrgeometer circuitry compensates for the temperature dependence of the calibration factor $\left(c_{1}\right.$ $+c_{2} T_{0}{ }^{3}$ ) and supplies a voltage output -equivalent to $\sigma T_{0}{ }^{4}$. Albrecht and Cox (1977) gave a detailed analysis of the behavior of these circuits.

When the dome and base temperatures are different, the performance of the pyrgeometer is described by

$$
\begin{aligned}
L=\left(c_{1}+c_{2} T_{0}{ }^{3}\right) & V+\sigma T_{0}{ }^{4} \\
& -\left[\left(\alpha_{0}+4 \epsilon_{0} \epsilon \sigma T_{0}{ }^{3}\right) / \epsilon_{0} \tau\right]\left(T-T_{0}\right) .
\end{aligned}
$$

This expression includes an additional term that accounts for the energy transferred by conduction and radiation between dome and base. An evaluation of this term for a temperature of $288 \mathrm{~K}$ shows that about $11-12 \mathrm{~W} \mathrm{~m}^{-2}$ are included in the measurements of the pyrgeometer for each degree of difference between the base and dome temperatures. This estimate is in good agreement with the experimental results of Berdahl and Fromberg (1982).

The pyrgeometer calibration was carried out using a blackbody cavity similar to that provided by Eppley. In these checks we followed the procedures of Albrecht et al. (1977). These checks had to be executed with special care, avoiding long exposures of the pyrgeometer to the blackbody cavity, as the radiative cooling of the filter dome may give rise to erroneous results. For this reason, in these checks we exposed the pyrgeometer to the blackbody cavity for 5 to $10 \mathrm{sec}$, during which time the radiometer output was stable.

The results of these checks indicated that the values of the calibration factor were always within $\pm 2.5 \%$ of the value indicated by Eppley (who used a blackbody accessory similar to the one we used). This is a good result according to the Eppley specification (1971), which deemed $\pm 3 \%$ as acceptable.

The fluctuations in the pyrgeometer output can be explained as a consequence of the combined influence of the solar heating effect over the dome [predicted by Eq. (2)] and of the presence of variable wind gusts. Due to the characteristics of the filter in the pyrgeometer dome, the solar radiation may make the difference ( $T$ $T_{0}$ ) greater than zero, thus yielding an additional energy flux [cf. Eq. (2)] over the sensor surface. When variable wind gusts are present, however, changes will occur in the dome temperature, thus provoking a variable or fluctuating output of the pyrgeometer.

The effect of solar heating of the dome on the instrument was evaluated by sun and shade experiments. These experiments consisted of observations made at different hours during cloudless days, using a disk held at a given distance over the radiometer to shade the instrument. We observed differences of $13-30 \mathrm{~W} \mathrm{~m}^{-2}$ between shaded and unshaded pyrgeometer measurements. These results agree with those of Berdahl et al. (1982) and Weiss (1981). They represent a significant improvement over the former KRS-5 pyrgeometer that, as Enz et al. (1975) observed, had a solar effect ranging between 20 and $120 \mathrm{~W} \mathrm{~m}^{-2}$. The response time of the pyrgeometer to changes due to shading the dome was determined to be about $30 \mathrm{sec}$, in accordance with the previous results of Berdahl and Fromberg (1982). The comparison between this time and the normal response time of the instrument $(2 \mathrm{sec})$ gives a clear indication that the solar effect on the pyrgeometer is not caused by direct transmission of solar radiation through the filter dome.

In order to eliminate the error associated with the solar heating of the dome, Enz et al. (1975) reduced the magnitude of the error to $50 \%$ by ventilating the dome. Albrecht and Cox (1977) proposed a correction based on the measurement of the dome temperature. Nevertheless, this is a difficult task, and one faces the problem that the temperature at a single point may not be representative of the whole dome; the problem being complicated by differential heating of the dome due to variations in solar geometry. Recently, Yamanouchi et al. (1984) used a shading ring to protect the pyrgeometer from solar radiation.

The purpose of this paper is an experimental study of the correlation between the error due to solar heating of the dome and its direct cause, solar radiation. For our experiments we use the pyrgeometer measurements and those of direct solar radiation supplied by the Middleton pyrheliometer mentioned previously.

\section{Results}

A set of sun and shade experiments have been made in several cloudless days with wind speeds below $1 \mathrm{~m}$ $\mathrm{s}^{-1}$. In these experiments the pyrgeometer dome was alternately shaded and unshaded by means of an $8 \mathrm{~cm}$ diameter disk. Taking into account the response time of the radiometer to the solar heating effect, the exposition periods were about $60 \mathrm{sec}$ so as to allow the pyrgeometer to reach a stable output. Because the viewing angle of the pryheliometer is about $5^{\circ}$, the disk was held at such a distance that the angle subtended by the disk from the pyrgeometer sensor was 


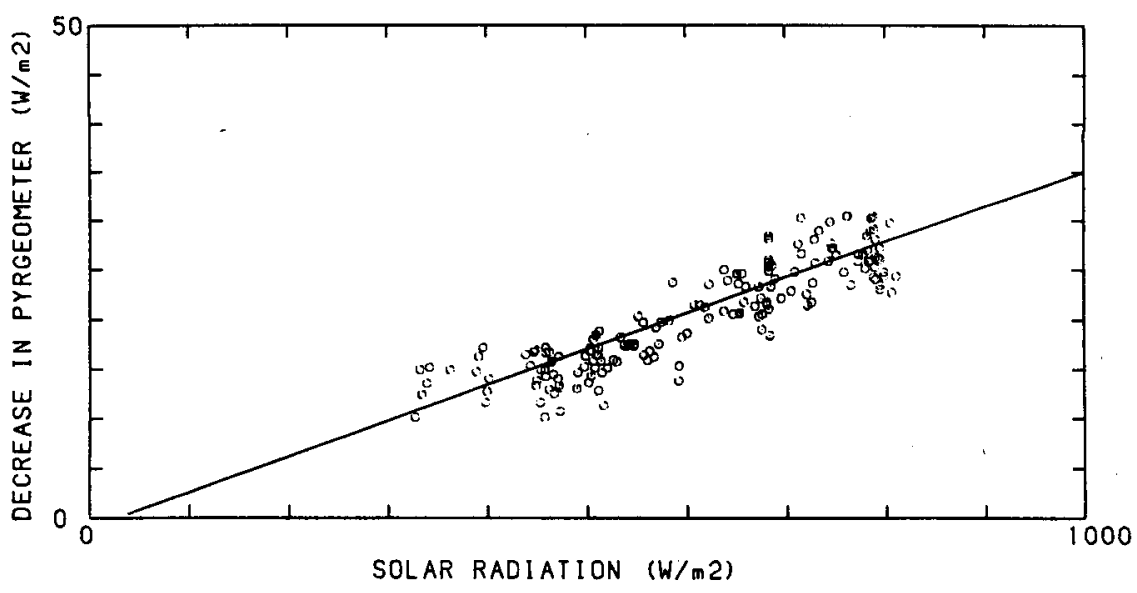

FIG. 1. Decrease in longwave irradiance measured with the pyrgeometer due to shading of the dome versus solar radiation measurements, for cloudless days with wind speeds lower than $1 \mathrm{~m} \mathrm{~s}^{-1}$.

also approximately $5^{\circ}$. Prior to these experiments, tests were made holding the shading disk at various positions around the unshaded pyrgeometer in order to ensure that radiation from the disk did not affect pyrgeometer readings.

The thermopile output was directly measured and the $\sigma T_{0}^{4}$ term was evaluated with simultaneous measurements of the pyrgeometer base temperature to avoid the error associated with the emission compensation circuit (Albrecht et al. 1977).

Results of the experiments are shown in Fig. 1. Experimental points correspond to instantaneous measurements, the solid line being the representation of

$$
\Delta L=-1.0+0.036 S\left[\mathrm{~W} \mathrm{~m}^{-2}\right],
$$

obtained by regression of the differences between shaded and unshaded pyrgeometer measurements, $\Delta L$, and the direct solar radiation on an horizontal surface, $S$. The correlation coefficient between $\Delta L$ and $S$ was calculated to be $r=0.899$. The scatter may be due to the experimental errors associated with the pyrgeometer and pyrheliometer measurements, which have been estimated to be around $1 \%-2 \%$. Equation (3) indicates that $3.6 \%$ of the solar flux incident on the pyrgeometer is included in its measurements. Thus, the maximum error produced by solar heating of the dome is about $10 \%$ of the thermal atmospheric radiation measurements obtained in our experiments. Such results are significantly better than those obtained with the former KRS-5 pyrgeometer, which includes about 9\% of the solar incident radiation (Enz et al. 1975).

In order to study the wind effect on the instrument, several experiments were conducted on cloudless days with wind speeds greater than $1 \mathrm{~m} \mathrm{~s}^{-1}$. The wind speed was determined with an anemometer placed near the radiometers. Figure 2 shows the results of these experiments. The experimental points correspond to different wind speed intervals. The solid line is that of Eq.

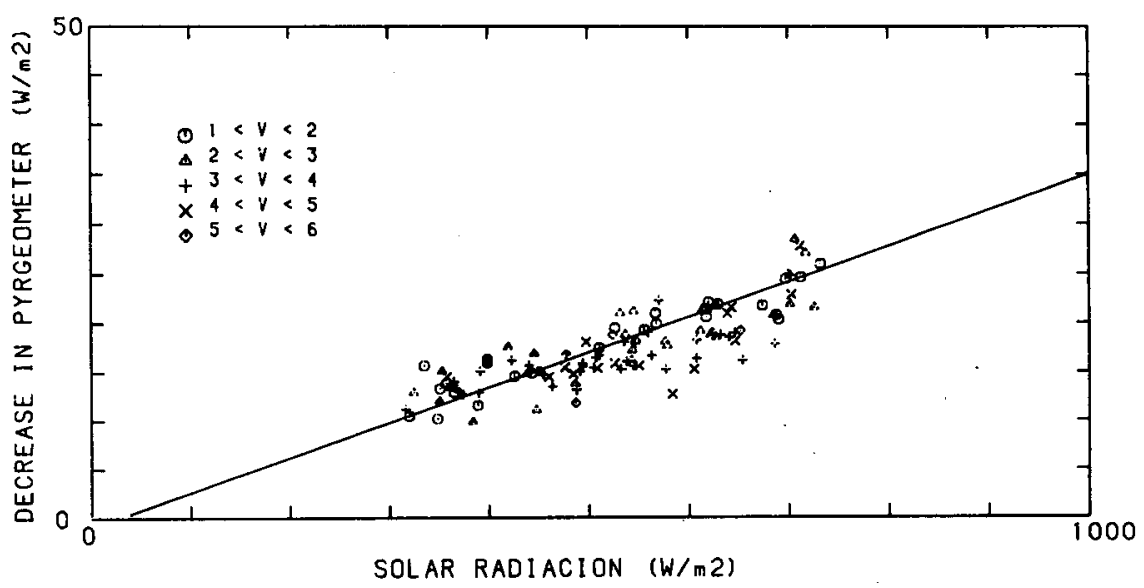

FIG. 2. As in Fig. 1 but with wind speeds greater than $1 \mathrm{~m} \mathrm{~s}^{-1}$. 
(3), obtained in the regression analysis of the experimental data with wind speeds below $1 \mathrm{~m} \mathrm{~s}^{-1}$. The figure shows that the heating effect over the dome is well explained by Eq. (3), in the presence of variable wind speeds. Only the experimental points associated with wind speeds greater than $4 \mathrm{~m} \mathrm{~s}^{-1}$ are not well explained by the regression line. These findings, together with the fact that an air flow as high as $13 \mathrm{~m} \mathrm{~s}^{-1}$ was needed by Enz et al. (1975) for obtaining a 50\% reduction in the heating effect, led us to consider that Eq. (3) may provide an acceptable correction to the solar heating effect over the pyrgeometer dome. We assume that the heating effect due to diffuse solar radiation is similar to that due to direct solar radiation. Hence, the correction term is evaluated using the global solar radiation instead of the direct solar radiation over a horizontal surface, $S$.

\section{Conclusions}

The results of this study indicate that measurements with the Eppley silicon dome pyrgeometer involve errors due to the solar heating effect on the dome. The magnitude of this error is lower than that associated with the former version of the pyrgeometer (with KRS5 filter), but may amount to about $10 \%$ of the measured thermal atmospheric radiation.

For similar reasons, the pyrgeometer calibration factor must be checked carefully, due to the problems associated with the temperature differences between the pyrgeometer base and dome caused by prolongated exposition to the blackbody cavity used.

In order to correct for the anomalous effect of solar heating, an error term evaluated from global solar radiation measurements is presented. This empirical relation provides an acceptable estimation even for a variety of wind speed conditions.

Acknowledgments. This research was supported by a grant from the CAICYT Institution, supported by the Spanish Education and Science Ministry. Valuable comments in the translation of this manuscript were provided by Dr. A. Delgado.

\section{APPENDIX}

\section{List of symbols}

$\epsilon_{0}$ Longwave emissivity of the sensor surface

$\rho_{0} \quad$ Longwave reflectivity of the sensor surface

$\epsilon \quad$ Longwave emissivity of the silicon dome

$\tau \quad$ Longwave transmissivity of the silicon dome

$\rho$ Longwave reflectivity of the dome

$T_{s} \quad$ Temperature of the sensor surface (K)

$T_{0} \quad$ Temperature of the pyrgeometer base $(\mathrm{K})$

$T$ Temperature of the silicon dome (K)

$\alpha \quad$ The effective heat transfer coefficient (conduction and convection by the surrounding gas) (W $\mathrm{m}^{-2}{ }^{\circ} \mathrm{C}^{-1}$ )

$\alpha_{0}$ The effective heat transfer coefficient due to con- duction by the air between dome and sensor

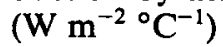

$\beta \quad$ The effective thermal conductivity (by metal and other materials) between the receiver and its environment $\left(\mathrm{W} \mathrm{m} \mathrm{m}^{-2}{ }^{\circ} \mathrm{C}^{-1}\right)$

$L \quad$ Thermal atmospheric irradiance at pyrgeometer level

$H$ Sensible heat flux of the sensor surface $\left(\mathrm{W} \mathrm{m}^{-2}\right)$

$\sigma \quad$ Stefan-Boltzmann constant

$V \quad$ Thermopile output (V)

\section{Sensor surface energy budget deduction}

Because of the filtering of the pyrgeometer dome, the energy budget over the sensor surface can be written as

$$
L_{i}-L_{0}=H
$$

where $L_{i}$ is the thermal irradiance incident upon the sensor surface and $L_{0}$ is the outgoing thermal irradiance from this same surface, obtained as

$$
\begin{aligned}
& L_{i}=\tau L+\epsilon \sigma T^{4}+\rho L_{0} \\
& L_{0}=\epsilon_{0} \sigma T_{s}^{4}+\rho_{0} L_{i} .
\end{aligned}
$$

The sensible heat flux has the following expression

$$
H=(\alpha+\beta)\left(T_{s}-T_{0}\right)+\alpha_{0}\left(T_{s}-T\right)
$$

where the second term takes into account the energy transfer by conduction through the air included between dome and sensor.

From (A2) and (A3) and taking into account that (1 $\left.-\rho \rho_{0}\right) \approx 1.0$, because $\rho_{0}<0.02$ and $\rho \approx 0.3-0.5$, it is easy to obtain

$$
\begin{aligned}
& (\alpha+\beta)\left(T_{s}-T_{0}\right)+\alpha_{0}\left(T_{s}-T\right) \\
& \quad=\left(1-\rho_{0}\right) \tau L+\left(1-\rho_{0}\right) \epsilon \sigma T^{4}-\epsilon_{0} \sigma T_{s}^{4}(1-\rho) .
\end{aligned}
$$

Considering that $T-T_{0} \ll T_{0}$ and $T_{s}-T_{0} \ll T_{0}$, we can write

$$
T^{4}=T_{0}^{4}+4 T_{0}^{3}\left(T-T_{0}\right)
$$

and similarly

$$
T_{s}^{4}=T_{0}^{4}+4 T_{0}^{3}\left(T_{s}-T_{0}\right) .
$$

Since the maximum temperature differences between the pyrgeometer sensor and base are on the order of $0.5^{\circ} \mathrm{C}$ we may substitute $T_{0}$ for $T_{s}$ in $\alpha_{0}\left(T_{s}-T\right)$, thus obtaining

$$
\begin{aligned}
& {\left[\begin{array}{l}
\left.\alpha+\beta+4 \epsilon_{0} \sigma T_{0}{ }^{3}(1-\rho)\right]\left(T_{s}-T_{0}\right) \\
=\epsilon_{0} \tau L-\epsilon_{0} \sigma T_{0}{ }^{4}(1-\epsilon-\rho) \\
\quad+\left(4 \epsilon_{0} \epsilon \sigma T_{0}{ }^{3}+\alpha_{0}\right)\left(T-T_{0}\right) .
\end{array}\right.}
\end{aligned}
$$

Taking into account the proportionality between the thermopile output and the temperature differences between cold and hot junctions, we have

$$
V=k\left(T_{s}-T_{0}\right)
$$


where $k$ is a constant factor depending on the number of thermocouple junctions and their characteristics.

Since $(1-\epsilon-\rho)=\tau$, we have

$$
\begin{aligned}
L=\{ & {\left.\left[\alpha+\beta+4 \epsilon_{0} \epsilon \sigma T_{0}{ }^{3}(1-\rho)\right] / k \epsilon_{0} \tau\right\} V } \\
& +\sigma T_{0}{ }^{4}-\left[\left(\alpha_{0}+4 \epsilon_{0} \epsilon \sigma T_{0}{ }^{3}\right) / \epsilon_{0} \tau\right]\left(T-T_{0}\right) .
\end{aligned}
$$

The pyrgeometer characteristics imply that $\alpha$ and $\beta$ have only a negligible thermal dependence. On the other hand, $\alpha$ is for the same reason, practically independent of wind speed. Hence, we can write

$$
\begin{aligned}
L=\left(c_{1}+c_{2} T_{0}{ }^{3}\right) V+\sigma T_{0}{ }^{4} \\
-\left[\left(\alpha_{0}+4 \epsilon_{0} \epsilon \sigma T_{0}{ }^{3}\right) / \epsilon_{0} \tau\right]\left(T-T_{0}\right) .
\end{aligned}
$$

The last term in Eq. (A8) gives the additional energy fluxes due to the pyrgeometer base-dome temperature differences. The conduction part of this term, $\left(\alpha_{0} / \epsilon_{0} \tau\right)(T$ $-T_{0}$ ), can be shown to be about 7-8 $\mathrm{W} \mathrm{m}^{-2}$ by an approximate evaluation of $\alpha_{0}$. Since $\tau=0.5$ and taking into account that $\epsilon \approx 0.2-0.4$, we find for $T_{0}$
$=288 \mathrm{~K}$, that the radiative part of the term, $\left(4 \epsilon_{0} \epsilon \sigma T_{0}^{3} / \epsilon_{0} \tau\right)\left(T-T_{0}\right)$, amounts to about $3-4 \mathrm{~W} \mathrm{~m}^{-2}$.

\section{REFERENCES}

Albrecht, B., and S. K. Cox, 1977: Procedures for improving pyrgeometer performance. J. Appl. Meteor., 46, 188-197.

-, M. Poellot and S. K. Cox, 1974: Pyrgeometer measurements from aircraft. Rev. Sci. Instrum., 45(1), 33-38.

Berdahl, P., and R. Fromberg, 1982: The thermal radiance of clear skies. Sol. Energy, 29(4), 299-314.

Enz, J. W., J. C. Klink and D. G. Baker, 1975: Solar radiation effects on pyrgeometer performance. J. Appl. Meteor., 14, 1297-1302.

Eppley Laboratory, Inc., 1971: Instrumentation for the measurements of components of solar and terrestrial radiation. Unpublished document by Eppley Laboratory, Newport, 31 pp.

_- 1976: Instrumentation for the measurements of components of solar and terrestrial radiation. Unpublished document by.Eppley Laboratory, Newport, 12 pp.

Weiss, A., 1981: On the performance of pyrgeometers with silicon domes. J. Appl. Meteor., 20(8), 962-965.

Yamanouchi, T., and S. Kawaguchi, 1984: Longwave radiation balance under a strong surface inversion in the katabatic wind zone, Antarctica. J. Geophys. Res., 89(D7), 11 771-11 778. 